\title{
On Pseudohyperbolical Smarandache Curves in Minkowski 3-Space
}

\author{
Esra Betul Koc Ozturk, ${ }^{1,2}$ Ufuk Ozturk, ${ }^{1,2}$ Kazim İlarslan, ${ }^{3}$ and Emilija Nešovićc ${ }^{4}$ \\ ${ }^{1}$ Department of Mathematics, Faculty of Sciences, University of Cankiri Karatekin, Cankiri 18100, Turkey \\ ${ }^{2}$ School of Mathematics \& Statistical Sciences, Arizona State University, Room PSA442, Tempe, AZ 85287-1804, USA \\ ${ }^{3}$ Department of Mathematics, Faculty of Sciences and Art, University of Kirlkkale, Kirıkkale 71450, Turkey \\ ${ }^{4}$ University of Kragujevac, Faculty of Science, Department of Mathematics and Informatics, Kragujevac 34000, Serbia
}

Correspondence should be addressed to Ufuk Ozturk; uuzturk@asu.edu

Received 10 March 2013; Accepted 7 May 2013

Academic Editor: B. N. Mandal

Copyright (C) 2013 Esra Betul Koc Ozturk et al. This is an open access article distributed under the Creative Commons Attribution License, which permits unrestricted use, distribution, and reproduction in any medium, provided the original work is properly cited.

We define pseudohyperbolical Smarandache curves according to the Sabban frame in Minkowski 3-space. We obtain the geodesic curvatures and the expression for the Sabban frame vectors of special pseudohyperbolic Smarandache curves. Finally, we give some examples of such curves.

\section{Introduction}

In the theory of curves in the Euclidean and Minkowski spaces, one of the interesting problems is the problem of characterization of a regular curve. In the solution of the problem, the curvature functions $\kappa$ and $\tau$ of a regular curve have an effective role. It is known that we can determine the shape and size of a regular curve by using its curvatures $\kappa$ and $\tau$. Another approach to the solution of the problem is to consider the relationship between the corresponding Frenet vectors of two curves. For instance, Bertrand curves and Mannheim curves arise from this relationship. Another example is the Smarandache curves. They are the objects of Smarandache geometry, that is, a geometry which has at least one Smarandachely denied axiom [1]. An axiom is said to be Smarandachely denied if it behaves in at least two different ways within the same space. Smarandache geometries are connected with the theory of relativity and the parallel universes.

If the position vector of a regular curve $\alpha$ is composed by the Frenet frame vectors of another regular curve $\beta$, then the curve $\alpha$ is called a Smarandache curve [2]. Special Smarandache curves in Euclidean and Minkowski spaces are studied by some authors [3-8]. The curves lying on a pseudohyperbolic space $H_{0}^{2}$ in Minkowski 3-space $\mathbb{E}_{1}^{3}$ are characterized in [9].

In this paper, we define pseudohyperbolical Smarandache curves according to the Sabban frame $\{\alpha, T, \xi\}$ in Minkowski 3 -space. We obtain the geodesic curvatures and the expressions for the Sabban frame's vectors of special pseudohyperbolical Smarandache curves. In particular, we prove that special $T \xi$-pseudohyperbolical Smarandache curves do not exist. Besides, we give some examples of special pseudohyperbolical Smarandache curves in Minkowski 3-space.

\section{Basic Concepts}

The Minkowski 3-space $\mathbb{R}_{1}^{3}$ is the Euclidean 3-space $\mathbb{R}^{3}$ provided with the standard flat metric given by

$$
\langle\cdot, \cdot\rangle=-d x_{1}^{2}+d x_{2}^{2}+d x_{3}^{2},
$$

where $\left(x_{1}, x_{2}, x_{3}\right)$ is a rectangular Cartesian coordinate system of $\mathbb{R}_{1}^{3}$. Since $g$ is an indefinite metric, recall that a nonzero vector $\vec{x} \in \mathbb{R}_{1}^{3}$ can have one of the three Lorentzian causal characters: it can be spacelike if $\langle\vec{x}, \vec{x}\rangle>0$, timelike if $\langle\vec{x}, \vec{x}\rangle<$ 0 , and null (lightlike) if $\langle\vec{x}, \vec{x}\rangle=0$. In particular, the norm (length) of a vector $\vec{x} \in \mathbb{R}_{1}^{3}$ is given by $\|\vec{x}\|=\sqrt{|\langle\vec{x}, \vec{x}\rangle|}$ and 
two vectors $\vec{x}$ and $\vec{y}$ are said to be orthogonal if $\langle\vec{x}, \vec{y}\rangle=0$. Next, recall that an arbitrary curve $\alpha=\alpha(s)$ in $\mathbb{E}_{1}^{3}$ can locally be spacelike, timelike, or null (lightlike) if all of its velocity vectors $\alpha^{\prime}(s)$ are, respectively, spacelike, timelike, or null (lightlike) for every $s \in I$ [10]. If $\left\|\alpha^{\prime}(s)\right\| \neq 0$ for every $s \in I$, then $\alpha$ is a regular curve in $\mathbb{R}_{1}^{3}$. A spacelike (timelike) regular curve $\alpha$ is parameterized by pseudo-arclength parameter $s$ which is given by $\alpha: I \subset \mathbb{R} \rightarrow \mathbb{R}_{1}^{3}$, and then the tangent vector $\alpha^{\prime}(s)$ along $\alpha$ has unit length, that is, $\left\langle\alpha^{\prime}(s), \alpha^{\prime}(s)\right\rangle=$ $1\left(\left\langle\alpha^{\prime}(s), \alpha^{\prime}(s)\right\rangle=-1\right)$ for all $s \in I$, respectively.

For any $\vec{x}=\left(x_{1}, x_{2}, x_{3}\right)$ and $\vec{y}=\left(y_{1}, y_{2}, y_{3}\right)$ in the space $\mathbb{R}_{1}^{3}$, the pseudovector product of $\vec{x}$ and $\vec{y}$ is defined by

$$
\vec{x} \times \vec{y}=\left(-x_{2} y_{3}+x_{3} y_{2}, x_{3} y_{1}-x_{1} y_{3}, x_{1} y_{2}-x_{2} y_{1}\right) .
$$

Remark 1. Let $\vec{x}=\left(x_{1}, x_{2}, x_{3}\right), \vec{y}=\left(y_{1}, y_{2}, y_{3}\right)$, and $\vec{z}=$ $\left(z_{1}, z_{2}, z_{3}\right)$ be vectors in $\mathbb{R}_{1}^{3}$. Then,

(i) $\langle\vec{x} \times \vec{y}, \vec{z}\rangle=\left|\begin{array}{lll}x_{1} & x_{2} & x_{3} \\ y_{1} & y_{2} & y_{3} \\ z_{1} & z_{2} & z_{3}\end{array}\right|$,

(ii) $\vec{x} \times(\vec{y} \times \vec{z})=-\langle\vec{x}, \vec{z}\rangle \vec{y}+\langle\vec{x}, \vec{y}\rangle \vec{z}$,

(iii) $\langle\vec{x} \times \vec{y}, \vec{x} \times \vec{y}\rangle=-\langle\vec{x}, \vec{x}\rangle\langle\vec{y}, \vec{y}\rangle+\langle\vec{x}, \vec{y}\rangle^{2}$,

where $\times$ is the pseudovector product in the space $\mathbb{R}_{1}^{3}$.

Lemma 2. In the Minkowski 3-space $\mathbb{R}_{1}^{3}$, the following properties are satisfied [10]:

(i) two timelike vectors are never orthogonal;

(ii) two null vectors are orthogonal if and only if they are linearly dependent;

(iii) timelike vector is never orthogonal to a null vector.

Pseudohyperbolic space in the Minkowski 3-space $\mathbb{R}_{1}^{3}$ is a quadric defined by

$$
H_{0}^{2}=\left\{\vec{x} \in \mathbb{R}_{1}^{3} \mid-x_{1}^{2}+x_{2}^{2}+x_{3}^{2}=-1\right\} .
$$

Let $\alpha: I \subset \mathbb{R} \rightarrow H_{0}^{2}$ be a regular unit speed curve lying fully in $H_{0}^{2}$ in $\mathbb{R}_{1}^{3}$. Then its position vector $\alpha$ is timelike vector, which implies that tangent vector $\vec{T}=\alpha^{\prime}$ is the unit spacelike vector for all $s \in I$. Hence we have orthonormal Sabban frame $\{\alpha(s), T(s), \xi(s)\}$ along the curve $\alpha$, where $\xi(s)=\alpha(s) \times T(s)$ is the unit spacelike vector. The corresponding Frenet formulae of $\alpha$, according to the Sabban frame, read

$$
\left[\begin{array}{l}
\alpha^{\prime} \\
T^{\prime} \\
\xi^{\prime}
\end{array}\right]=\left[\begin{array}{ccc}
0 & 1 & 0 \\
1 & 0 & k_{g}(s) \\
0 & -k_{g}(s) & 0
\end{array}\right]\left[\begin{array}{l}
\alpha \\
T \\
\xi
\end{array}\right],
$$

where $k_{g}(s)=\operatorname{det}\left(\alpha(s), T(s), T^{\prime}(s)\right)$ is the geodesic curvature of $\alpha$ on $H_{0}^{2}$ in $\mathbb{R}_{1}^{3}$ and $s$ is arc length parameter of alpha. In particular, the following relations hold:

$$
\alpha \times T=\xi, \quad T \times \xi=-\alpha, \quad \xi \times \alpha=T .
$$

\section{Pseudohyperbolical Smarandache Curves in Minkowski 3-Space}

In this section, we define and investigate pseudohyperbolical Smarandache curves in Minkowski 3-space according to the Sabban frame.

Let $\alpha=\alpha(s)$ and $\beta=\beta\left(s^{*}\right)$ be two regular unit speed curves lying fully in pseudohyperbolic space $H_{0}^{2}$ in $\mathbb{R}_{1}^{3}$ and let $\{\alpha, T, \xi\}$ and $\left\{\beta, T_{\beta}, \xi_{\beta}\right\}$ be the moving Sabban frames of these curves, respectively. Then we have the following definitions of pseudohyperbolical Smarandache curves.

Definition 3. Let $\alpha: I \subset \mathbb{R} \mapsto H_{0}^{2}$ be a regular unit speed curve lying fully in $H_{0}^{2}$. Then $\alpha \xi$-pseudohyperbolical Smarandache curve $\beta: I \subset \mathbb{R} \mapsto H_{0}^{2}$ of $\alpha$ is defined by

$$
\beta\left(s^{\star}(s)\right)=\frac{1}{\sqrt{2}}(a \alpha(s)+b \xi(s)),
$$

where $a, b \in \mathbb{R}_{0}$ and $a^{2}-b^{2}=2$.

Definition 4. Let $\alpha: I \subset \mathbb{R} \mapsto H_{0}^{2}$ be a regular unit speed curve lying fully in $H_{0}^{2}$.Then $\alpha T$-pseudohyperbolical Smarandache curve $\beta: I \subset \mathbb{R} \mapsto H_{0}^{2}$ of $\alpha$ is defined by

$$
\beta\left(s^{\star}(s)\right)=\frac{1}{\sqrt{2}}(a \alpha(s)+b T(s)),
$$

where $a, b \in R_{0}$ and $a^{2}-b^{2}=2$.

Definition 5. Let $\alpha: I \subset R \mapsto H_{0}^{2}$ be a regular unit speed curve lying fully in $H_{0}^{2}$. Then $\alpha T \xi$-pseudohyperbolical Smarandache curve $\beta: I \subset R \mapsto H_{0}^{2}$ of $\alpha$ is defined by

$$
\beta\left(s^{\star}(s)\right)=\frac{1}{\sqrt{3}}(a \alpha(s)+b T(s)+c \xi(s)),
$$

where $a, b, c \in \mathbb{R}_{0}$ and $a^{2}-b^{2}-c^{2}=3$.

Theorem 6. Let $\alpha: I \subset \mathbb{R} \mapsto H_{0}^{2}$ be a regular unit speed curve lying fully in $H_{0}^{2}$. Then $T \xi$-pseudohyperbolical Smarandache curve $\beta: I \subset \mathbb{R} \mapsto H_{0}^{2}$ of $\alpha$ does not exist.

Proof. Assume that there exists $T \xi$-pseudohyperbolical Smarandache curve of $\alpha$. Then it can be written as

$$
\beta\left(s^{\star}(s)\right)=\frac{1}{\sqrt{2}}(a T(s)+b \xi(s)),
$$

where $a^{2}+b^{2}=-2$, which is a contradiction.

In the theorems which follow, we obtain Sabban frame $\left\{\beta, T_{\beta}, \xi_{\beta}\right\}$ and geodesic curvature $\kappa_{g}^{\beta}$ of pseudohyperbolical Smarandache curve $\beta$.

Theorem 7. Let $\alpha: I \subset \mathbb{R} \mapsto H_{0}^{2}$ be a regular unit speed curve lying fully in $H_{0}^{2}$ with the Sabban frame $\{\alpha, T, \xi\}$ and 
the geodesic curvature $k_{g}$. If $\beta: I \subset \mathbb{R} \mapsto H_{0}^{2}$ is $\alpha \xi$ pseudohyperbolical Smarandache curve of $\alpha$, then its frame $\left\{\beta, T_{\beta}, \xi_{\beta}\right\}$ is given by

$$
\left[\begin{array}{c}
\beta \\
T_{\beta} \\
\xi_{\beta}
\end{array}\right]=\left[\begin{array}{ccc}
\frac{a}{\sqrt{2}} & 0 & \frac{b}{\sqrt{2}} \\
0 & \epsilon & 0 \\
\epsilon \frac{b}{\sqrt{2}} & 0 & \epsilon \frac{a}{\sqrt{2}}
\end{array}\right]\left[\begin{array}{l}
\alpha \\
T \\
\xi
\end{array}\right],
$$

and the corresponding geodesic curvature $k_{g}^{\beta}$ reads

$$
k_{g}^{\beta}=\frac{a k_{g}-b}{\left|a-b k_{g}\right|}, \quad a, b \in \mathbb{R}_{0},
$$

where $a^{2}-b^{2}=2$ and $\epsilon= \pm 1$.

Proof. Differentiating (6) with respect to $s$ and using (4), we obtain

$$
\begin{gathered}
\beta^{\prime}(s)=\frac{d \beta}{d s^{*}} \frac{d s^{*}}{d s}=\frac{a-b k_{g}}{\sqrt{2}} T, \\
T_{\beta} \frac{d s^{*}}{d s}=\frac{a-b k_{g}}{\sqrt{2}} T,
\end{gathered}
$$

where

$$
\frac{d s^{*}}{d s}=\frac{\left|a-b k_{g}\right|}{\sqrt{2}} .
$$

Therefore, the unit spacelike tangent vector of the curve $\beta$ is given by

$$
T_{\beta}=\epsilon T,
$$

where $\epsilon=+1$ if $a-b k_{g}>0$ for all $s$ and $\epsilon=-1$ if $a-b k_{g}<0$ for all $s$.

Differentiating (14) with respect to $s$, we find

$$
\frac{d T_{\beta}}{d s^{*}} \frac{d s^{*}}{d s}=\epsilon\left(\alpha+k_{g} \xi\right),
$$

and from (13) and (15) we get

$$
T_{\beta}^{\prime}=\frac{\sqrt{2} \epsilon}{\left|a-b k_{g}\right|}\left(\alpha+k_{g} \xi\right) .
$$
that

On the other hand, from (6) and (14) it can be easily seen

$$
\begin{aligned}
\xi_{\beta} & =\beta \times T_{\beta} \\
& =\epsilon \frac{b}{\sqrt{2}} \alpha+\epsilon \frac{a}{\sqrt{2}} \xi
\end{aligned}
$$

is a unit spacelike vector.

Consequently, the geodesic curvature $k_{g}^{\beta}$ of the curve $\beta=$ $\beta\left(s^{*}\right)$ is given by

$$
\begin{aligned}
k_{g}^{\beta} & =\operatorname{det}\left(\beta, T_{\beta}, T_{\beta}^{\prime}\right) \\
& =\frac{a k_{g}-b}{\left|a-b k_{g}\right|} .
\end{aligned}
$$

Theorem 8. Let $\alpha: I \subset \mathbb{R} \mapsto H_{0}^{2}$ be a regular unit speed curve lying fully in $H_{0}^{2}$ with the Sabban frame $\{\alpha, T, \xi\}$ and the geodesic curvature $k_{g}$. If $\beta: I \subset \mathbb{R} \mapsto H_{0}^{2}$ is $\alpha T$ pseudohyperbolical Smarandache curve of $\alpha$, then its frame $\left\{\beta, T_{\beta}, \xi_{\beta}\right\}$ is given by

$$
\left[\begin{array}{c}
\beta \\
T_{\beta} \\
\xi_{\beta}
\end{array}\right]
$$

$$
\begin{aligned}
& {\left[\begin{array}{ccc}
\frac{a}{\sqrt{2}} & \frac{b}{\sqrt{2}} & 0 \\
\frac{b}{\sqrt{2+\left(b k_{g}\right)^{2}}} & \frac{a}{\sqrt{2+\left(b k_{g}\right)^{2}}} & \frac{b k_{g}}{\sqrt{2+\left(b k_{g}\right)^{2}}} \\
-\frac{b^{2} k_{g}}{\sqrt{4+2\left(b k_{g}\right)^{2}}} & -\frac{a b k_{g}}{\sqrt{4+2\left(b k_{g}\right)^{2}}} & \frac{\sqrt{2}}{\sqrt{2+\left(b k_{g}\right)^{2}}}
\end{array}\right]} \\
& \times\left[\begin{array}{c}
\alpha \\
T \\
\xi
\end{array}\right],
\end{aligned}
$$

and the corresponding geodesic curvature $k_{g}^{\beta}$ reads

$$
k_{g}^{\beta}=\frac{b^{2} k_{g} \varepsilon_{1}-a b k_{g} \varepsilon_{2}+2 \varepsilon_{3}}{\left(2+b^{2} k_{g}^{2}\right)^{5 / 2}}, \quad a, b \in \mathbb{R}_{0},
$$

where

$$
\begin{gathered}
\varepsilon_{1}=-b^{3} k_{g} k_{g}^{\prime}+a\left(2+\left(b k_{g}\right)^{2}\right), \\
\varepsilon_{2}=-a b^{2} k_{g} k_{g}^{\prime}+\left(b-b k_{g}^{2}\right)\left(2+\left(b k_{g}\right)^{2}\right), \\
\varepsilon_{3}=-b^{3} k_{g}^{2} k_{g}^{\prime}+\left(a k_{g}+b k_{g}^{\prime}\right)\left(2+\left(b k_{g}\right)^{2}\right),
\end{gathered}
$$

and $a^{2}-b^{2}=2$

Proof. Differentiating (7) with respect to $s$ and using (4), we obtain

$$
\begin{gathered}
\beta^{\prime}(s)=\frac{d \beta}{d s^{*}} \frac{d s^{*}}{d s}=\frac{1}{\sqrt{2}}\left(b \alpha+a T+b k_{g} \xi\right), \\
T_{\beta} \frac{d s^{*}}{d s}=\frac{1}{\sqrt{2}}\left(b \alpha+a T+b k_{g} \xi\right),
\end{gathered}
$$

where

$$
\frac{d s^{*}}{d s}=\sqrt{\frac{2+\left(b k_{g}\right)^{2}}{2}} .
$$

Therefore, the unit spacelike tangent vector of the curve $\beta$ is given by

$$
T_{\beta}=\frac{1}{\sqrt{2+\left(b k_{g}\right)^{2}}}\left(b \alpha+a T+b k_{g} \xi\right) .
$$


Differentiating (24) with respect to $s$, it follows that

$$
\frac{d T_{\beta}}{d s^{*}} \frac{d s^{*}}{d s}=\frac{1}{\left(2+\left(b k_{g}\right)^{2}\right)^{3 / 2}}\left(\varepsilon_{1} \alpha+\varepsilon_{2} T+\varepsilon_{3} \xi\right) .
$$

From (4) and (23), we get

$$
T_{\beta}^{\prime}=\frac{\sqrt{2}}{\left(2+\left(b k_{g}\right)^{2}\right)^{2}}\left(\varepsilon_{1} \alpha+\varepsilon_{2} T+\varepsilon_{3} \xi\right),
$$

where

$$
\begin{gathered}
\varepsilon_{1}=-b^{3} k_{g} k_{g}^{\prime}+a\left(2+\left(b k_{g}\right)^{2}\right), \\
\varepsilon_{2}=-a b^{2} k_{g} k_{g}^{\prime}+\left(b-b k_{g}^{2}\right)\left(2+\left(b k_{g}\right)^{2}\right), \\
\varepsilon_{3}=-b^{3} k_{g}^{2} k_{g}^{\prime}+\left(a k_{g}+b k_{g}^{\prime}\right)\left(2+\left(b k_{g}\right)^{2}\right) .
\end{gathered}
$$

$$
\left[\begin{array}{c}
\beta \\
T_{\beta} \\
\xi_{\beta}
\end{array}\right]=\left[\begin{array}{ccc}
\frac{a}{\sqrt{3}} & \frac{b}{\sqrt{3}} & \frac{c}{\sqrt{3}} \\
\frac{b}{\sqrt{\left|\left(a k_{g}-c\right)^{2}-3 k_{g}^{2}+3\right|}} & \frac{a-c k_{g}}{\sqrt{\left|\left(a k_{g}-c\right)^{2}-3 k_{g}^{2}+3\right|}} & \frac{b k_{g}}{\sqrt{\left|\left(a k_{g}-c\right)^{2}-3 k_{g}^{2}+3\right|}} \\
\frac{-\left(a^{2}-3\right) k_{g}+a c}{\sqrt{\left|3\left(a k_{g}-c\right)^{2}-9 k_{g}^{2}+9\right|}} & \frac{b c-a b k_{g}}{\sqrt{\left|3\left(a k_{g}-c\right)^{2}-9 k_{g}^{2}+9\right|}} & \frac{3+c^{2}-a c k_{g}}{\sqrt{\left|3\left(a k_{g}-c\right)^{2}-9 k_{g}^{2}+9\right|}}
\end{array}\right]\left[\begin{array}{l}
\alpha \\
T \\
\xi
\end{array}\right]
$$

and the corresponding geodesic curvature $k_{g}^{\beta}$ reads

$$
\begin{gathered}
k_{g}^{\beta}=\left(-\left(a c-\left(a^{2}-3\right) k_{g}\right) \varepsilon_{1}\right. \\
\left.+\left(b c-a b k_{g}\right) \varepsilon_{2}+\left(3+c^{2}-a c k_{g}\right) \varepsilon_{3}\right) \\
\times\left(\left(\left(a k_{g}-c\right)^{2}-3 k_{g}^{2}+3\right)^{5 / 2}\right)^{-1}, \\
a, b, c \in \mathbb{R}_{0},
\end{gathered}
$$

where

$$
\begin{aligned}
\varepsilon_{1}= & -\left(\left(a k_{g}-c\right) a k_{g}^{\prime}-3 k_{g}^{\prime} k_{g}\right) b \\
& +\left(\left(a k_{g}-c\right)^{2}-3 k_{g}^{2}+3\right)\left(a-c k_{g}\right),
\end{aligned}
$$

Theorem 9. Let $\alpha: I \subset \mathbb{R} \mapsto H_{0}^{2}$ be a regular unit speed curve lying fully in $H_{0}^{2}$ with the Sabban frame $\{\alpha, T, \xi\}$ and the geodesic curvature $k_{g}$. If $\beta: I \subset \mathbb{R} \mapsto H_{0}^{2}$ is $\alpha \mathrm{T} \xi$ pseudohyperbolical Smarandache curve of $\alpha$, then its frame $\left\{\beta, T_{\beta}, \xi_{\beta}\right\}$ is given by

$$
\begin{aligned}
\varepsilon_{2}= & -\left(\left(a k_{g}-c\right) a k_{g}^{\prime}-3 k_{g}^{\prime} k_{g}\right)\left(a-c k_{g}\right) \\
& +\left(\left(a k_{g}-c\right)^{2}-3 k_{g}^{2}+3\right)\left(b-c k_{g}^{\prime}-b k_{g}^{2}\right), \\
\varepsilon_{3}= & -\left(\left(a k_{g}-c\right) a k_{g}^{\prime}-3 k_{g}^{\prime} k_{g}\right) b k_{g} \\
& +\left(\left(a k_{g}-c\right)^{2}-3 k_{g}^{2}+3\right)\left(\left(a-c k_{g}\right) k_{g}+b k_{g}^{\prime}\right)
\end{aligned}
$$

Hence $\xi_{\beta}$ is a unit spacelike vector

Therefore, the geodesic curvature $k_{g}^{\beta}$ of the curve $\beta=$ $\beta\left(s^{*}\right)$ is given by

$$
\begin{aligned}
k_{g}^{\beta} & =\operatorname{det}\left(\beta, T_{\beta}, T_{\beta}^{\prime}\right) \\
& =\frac{b^{2} k_{g} \varepsilon_{1}-a b k_{g} \varepsilon_{2}+2 \varepsilon_{3}}{\left(2+b^{2} k_{g}^{2}\right)^{5 / 2}} .
\end{aligned}
$$

and $a^{2}-b^{2}-c^{2}=3$.

Proof. Differentiating (8) with respect to $s$ and by using (4), we find

$$
\beta^{\prime}(s)=\frac{d \beta}{d s^{*}} \frac{d s^{*}}{d s}=\frac{1}{\sqrt{3}}\left(b \alpha+\left(a-c k_{g}\right) T+b k_{g} \xi\right),
$$


and thus

$$
T_{\beta} \frac{d s^{*}}{d s}=\frac{1}{\sqrt{3}}\left(b \alpha+\left(a-c k_{g}\right) T+b k_{g} \xi\right),
$$

where

$$
\frac{d s^{*}}{d s}=\sqrt{\frac{\left|\left(a k_{g}-c\right)^{2}-3 k_{g}^{2}+3\right|}{3}} .
$$

The geodesic curvature $k_{g} \neq(a c \quad \pm$ $\left.\sqrt{a^{2} c^{2}-\left(a^{2}-3\right)\left(c^{2}+3\right)}\right) /\left(a^{2}-3\right)$ for all $s$, since $\beta=\beta\left(s^{*}\right)$ is a unit speed regular curve in $\mathbb{R}_{1}^{3}$.

Therefore, the unit spacelike tangent vector of the curve $\beta$ is given by

$$
T_{\beta}=\frac{1}{\sqrt{\left|\left(a k_{g}-c\right)^{2}-3 k_{g}^{2}+3\right|}}\left(b \alpha+\left(a-c k_{g}\right) T+b k_{g} \xi\right) .
$$

Differentiating (36) with respect to $s$ and from (4) and (35), it follows that

$$
T_{\beta}^{\prime}=\frac{\sqrt{3}}{\left(\left(a k_{g}-c\right)^{2}-3 k_{g}^{2}+3\right)^{2}}\left(\varepsilon_{1} \alpha+\varepsilon_{2} T+\varepsilon_{3} \xi\right),
$$

where

$$
\begin{aligned}
\varepsilon_{1}= & -\left(\left(a k_{g}-c\right) a k_{g}^{\prime}-3 k_{g}^{\prime} k_{g}\right) b \\
& +\left(\left(a k_{g}-c\right)^{2}-3 k_{g}^{2}+3\right)\left(a-c k_{g}\right), \\
\varepsilon_{2}= & -\left(\left(a k_{g}-c\right) a k_{g}^{\prime}-3 k_{g}^{\prime} k_{g}\right)\left(a-c k_{g}\right) \\
& +\left(\left(a k_{g}-c\right)^{2}-3 k_{g}^{2}+3\right)\left(b-c k_{g}^{\prime}-b k_{g}^{2}\right), \\
\varepsilon_{3}= & -\left(\left(a k_{g}-c\right) a k_{g}^{\prime}-3 k_{g}^{\prime} k_{g}\right) b k_{g} \\
& +\left(\left(a k_{g}-c\right)^{2}-3 k_{g}^{2}+3\right)\left(\left(a-c k_{g}\right) k_{g}+b k_{g}^{\prime}\right) .
\end{aligned}
$$
that

On the other hand, from (8) and (36) it can be easily seen

$$
\begin{aligned}
\xi_{\beta}= & \beta \times T_{\beta} \\
= & \frac{-\left(a^{2}-3\right) k_{g}+a c}{\sqrt{\left|3\left(a k_{g}-c\right)^{2}-9 k_{g}^{2}+9\right|}} \alpha \\
& +\frac{b c-a b k_{g}}{\sqrt{\left|3\left(a k_{g}-c\right)^{2}-9 k_{g}^{2}+9\right|}} T \\
& +\frac{3+c^{2}-a c k_{g}}{\sqrt{\left|3\left(a k_{g}-c\right)^{2}-9 k_{g}^{2}+9\right|}} \xi .
\end{aligned}
$$

Hence $\xi_{\beta}$ is a unit spacelike vector.

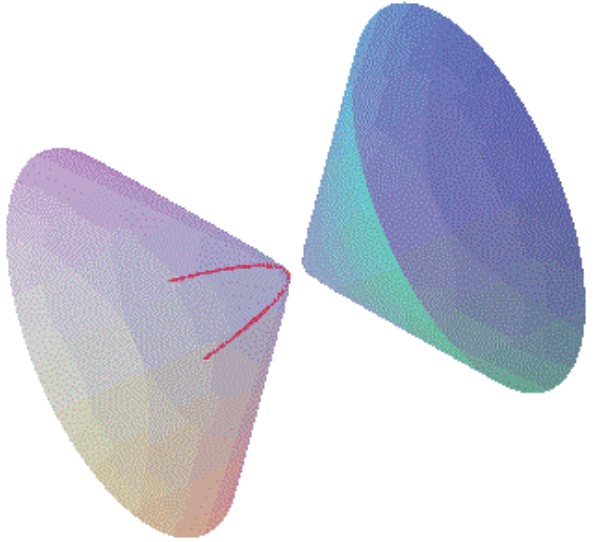

Figure 1: The curve $\alpha$ on $H_{0}^{2}(1)$.

Therefore, the geodesic curvature $k_{g}^{\beta}$ of the curve $\beta=$ $\beta\left(s^{*}\right)$ is given by

$$
\begin{aligned}
k_{g}^{\beta}= & \operatorname{det}\left(\beta, T_{\beta}, T_{\beta}^{\prime}\right) \\
= & \left(-\left(a c-\left(a^{2}-3\right) k_{g}\right) \varepsilon_{1}+\left(b c-a b k_{g}\right) \varepsilon_{2}\right. \\
& \left.+\left(3+c^{2}-a c k_{g}\right) \varepsilon_{3}\right) \\
& \times\left(\left(\left(a k_{g}-c\right)^{2}-3 k_{g}^{2}+3\right)^{5 / 2}\right)^{-1} .
\end{aligned}
$$

Corollary 10. If $\alpha: I \subset \mathbb{R} \mapsto H_{0}^{2}$ is a geodesic curve on $H_{0}^{2}$ in Minkowski 3-space $\mathbb{E}_{1}^{3}$, then

(1) $\alpha T$-pseudohyperbolic Smarandache curve is also geodesic on $H_{0}^{2}$;

(2) $\alpha \xi$-pseudohyperbolic and $\alpha T \xi$-pseudohyperbolic Smarandache curves have constant geodesic curvatures on $H_{0}^{2}$

\section{Examples}

Example 1. Let $\alpha$ be a unit speed curve lying in pseudohyperbolic space $H_{0}^{2}(1)$ in the Minkowski 3-space $\mathbb{E}_{1}^{3}$ with parameter equation (see Figure 1)

$$
\alpha(s)=\left(\frac{s^{2}}{2}+1, \frac{s^{2}}{2}, s\right) .
$$




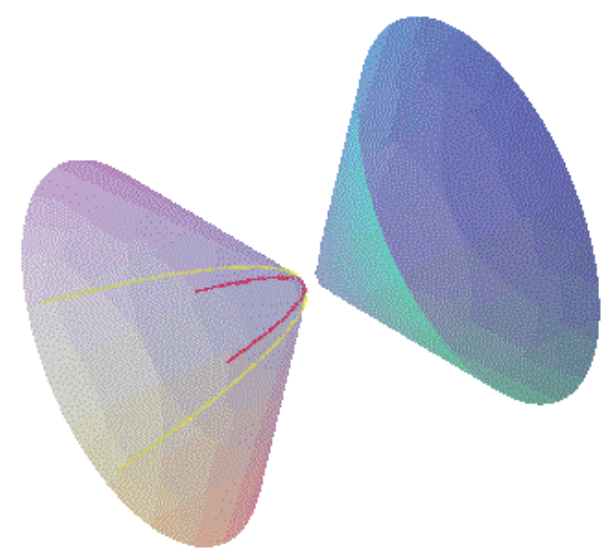

FIGURE 2: The $\alpha \xi$-pseudohyperbolical Smarandache curve $\beta$ and the curve $\alpha$ on $H_{0}^{2}(1)$.

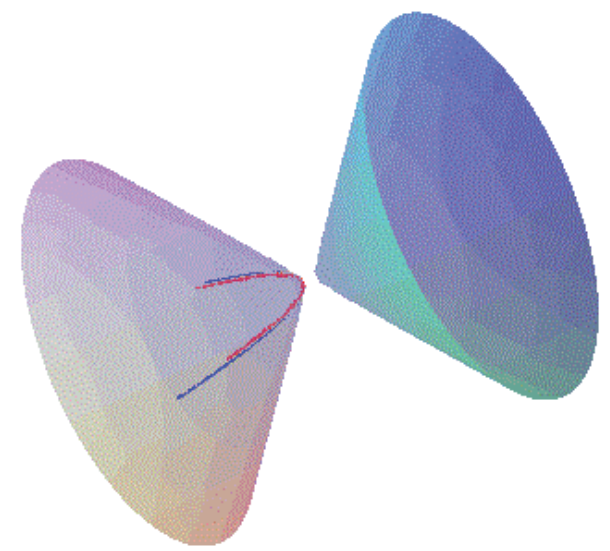

FIgURE 3: The $\alpha T$-pseudohyperbolical Smarandache curve $\beta$ and the curve $\alpha$ on $H_{0}^{2}(1)$.

The orthonormal Sabban frame $\{\alpha(s), T(s), \xi(s)\}$ along the curve $\alpha$ is given by

$$
\begin{gathered}
\alpha(s)=\left(\frac{s^{2}}{2}+1, \frac{s^{2}}{2}, s\right), \\
T(s)=\alpha^{\prime}(s)=(s, s, 1), \\
\xi(s)=\alpha(s) \times T(s)=\left(\frac{s^{2}}{2}, \frac{s^{2}}{2}-1, s\right) .
\end{gathered}
$$

In particular, the geodesic curvature $k_{g}$ of the curve $\alpha$ has the form

$$
k_{g}(s)=-1 \text {. }
$$

Case 1. If we take $a=2, b=\sqrt{2}$, then from (6) the $\alpha \xi$ pseudohyperbolical Smarandache curve $\beta$ is given by (see Figure 2)

$$
\begin{aligned}
\beta\left(s^{*}(s)\right)=\frac{1}{2} & \left((\sqrt{2}+1) s^{2}+2 \sqrt{2},\right. \\
& \left.(\sqrt{2}+1) s^{2}-2,(2 \sqrt{2}+2) s\right) .
\end{aligned}
$$

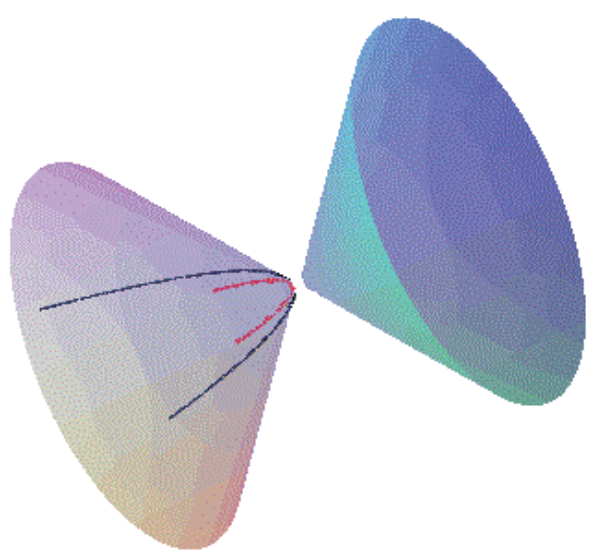

FIGURE 4: The $\alpha T \xi$-pseudohyperbolical Smarandache curve $\beta$ and the curve $\alpha$ on $H_{0}^{2}(1)$.

From Theorem 7 , its frame $\left\{\beta, T_{\beta}, \xi_{\beta}\right\}$ is given by

$$
\left[\begin{array}{c}
\beta \\
T_{\beta} \\
\xi_{\beta}
\end{array}\right]=\left[\begin{array}{ccc}
\sqrt{2} & 0 & 1 \\
0 & 1 & 0 \\
-1 & 0 & \sqrt{2}
\end{array}\right]\left[\begin{array}{l}
\alpha \\
T \\
\xi
\end{array}\right]
$$

and the corresponding geodesic curvature $k_{g}^{\beta}$ reads

$$
k_{g}^{\beta}=-1
$$

Case 2. If we take $a=\sqrt{3}, b=-1$, then from (7) the $\alpha T$-pseudohyperbolical Smarandache curve is given by (see Figure 3)

$$
\beta\left(s^{*}(s)\right)=\frac{\sqrt{2}}{4}\left(\sqrt{3} s^{2}-2 s+2 \sqrt{3}, \sqrt{3} s^{2}-2 s, 2 \sqrt{3} s-2\right) .
$$

According to Theorem 8 , its frame $\left\{\beta, T_{\beta}, \xi_{\beta}\right\}$ is given by

$$
\left[\begin{array}{c}
\beta \\
T_{\beta} \\
\xi_{\beta}
\end{array}\right]=\left[\begin{array}{ccc}
\frac{\sqrt{6}}{2} & -\frac{\sqrt{2}}{2} & 0 \\
-\frac{\sqrt{3}}{3} & 1 & \frac{\sqrt{3}}{3} \\
\frac{\sqrt{6}}{6} & -\frac{\sqrt{2}}{2} & \frac{\sqrt{6}}{3}
\end{array}\right]\left[\begin{array}{l}
\alpha \\
T \\
\xi
\end{array}\right],
$$

and the corresponding geodesic curvature $k_{g}^{\beta}$ reads

$$
k_{g}^{\beta}=-1 .
$$

Case 3. If $a=3, b=\sqrt{3}$, and $c=\sqrt{3}$, then from (7) the $\alpha T \xi$ pseudohyperbolical Smarandache curve $\beta$ is given by (see Figure 4)

$$
\begin{aligned}
\beta\left(s^{*}(s)\right)=\frac{1}{2}( & (\sqrt{3}+1) s^{2}+2 s+2 \sqrt{3}, \\
& \left.(\sqrt{3}+1) s^{2}+2 s-2,(2 \sqrt{3}+2) s+2\right) .
\end{aligned}
$$




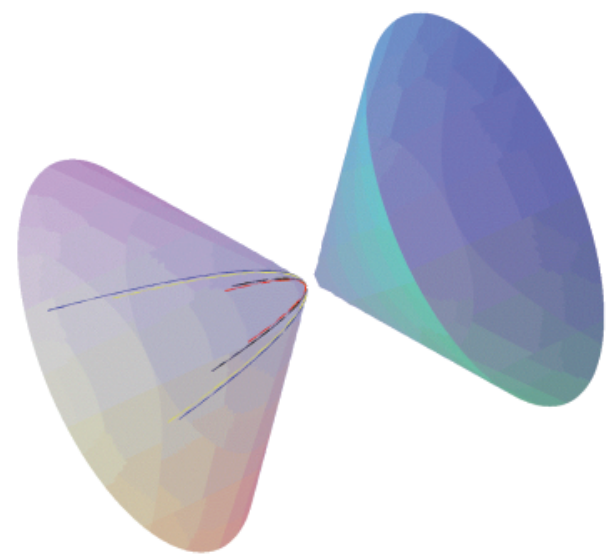

Figure 5: Pseudohyperbolical Smarandache curves of $\alpha$ and the curve $\alpha$ on $H_{0}^{2}(1)$.

By using Theorem 9, it follows that the frame $\left\{\beta, T_{\beta}, \xi_{\beta}\right\}$ is given by (see Figure 4)

$$
\left[\begin{array}{c}
\beta \\
T_{\beta} \\
\xi_{\beta}
\end{array}\right]=\left[\begin{array}{ccc}
\sqrt{3} & 1 & 1 \\
\frac{\sqrt{3}-1}{2} & 1 & \frac{1-\sqrt{3}}{2} \\
\frac{\sqrt{3}+1}{2} & 1 & \frac{\sqrt{3}+1}{2}
\end{array}\right]\left[\begin{array}{l}
\alpha \\
T \\
\xi
\end{array}\right]
$$

and the corresponding geodesic curvature $k_{g}^{\beta}$ reads

$$
k_{g}^{\beta}=-6(2+\sqrt{3})(3+\sqrt{3})^{1 / 2} .
$$

Also, Pseudohyperbolical Smarandache curves of $\alpha$ and the curve $\alpha$ on $H_{0}^{2}(1)$ with Figure 5 are shown.

\section{Acknowledgment}

The authors would like to thank the referees for their helpful suggestions and comments which significantly improved the first version of the paper.

\section{References}

[1] C. Ashbacher, "Smarandache geometries," Smarandache Notions Journal, vol. 8, no. 1-3, pp. 212-215, 1997.

[2] M. Turgut and S. Yilmaz, "Smarandache curves in Minkowski space-time," International Journal of Mathematical Combinatorics, vol. 3, pp. 51-55, 2008.

[3] A. T. Ali, "Special smarandache curves in the euclidean space," International Journal of Mathematical Combinatorics, vol. 2, pp. 30-36, 2010.

[4] K. Taşköprü and M. Tosun, "Smarandache curves on $S^{2}$," Boletim da Sociedade Paranaense de Matemática, vol. 32, no. 1, pp. 51-59, 2014.

[5] N. Bayrak, O. Bektas, and S. Yuce, "Special Smarandache a Curves in $\mathbb{E}_{1}^{3}$," In press, http://arxiv.org/abs/1204.5656.
[6] T. Körpinar and E. Turhan, "A new approach on timelike biharmonic slant helices according to Bishop frame in Lorentzian Heisenberg group Heis ${ }^{3}$," Kochi Journal of Mathematics, vol. 6, pp. 8-15, 2011.

[7] T. Körpinar and E. Turhan, "Characterization of Smarandache $M_{1} M_{2}$-curves of spacelike biharmonic $B$-slant helices according to Bishop frame in $\mathbb{E}(1,1)$," Advanced Modeling and Optimization, vol. 14, no. 2, pp. 327-333, 2012.

[8] Y. Yayli and E. Ziplar, "Frenet-serret motion and ruled surfaces with constant slope," International Journal of Physical Sciences, vol. 6, no. 29, pp. 6727-6734, 2011.

[9] M. Petrović-Torgašev and E. Šućurović, "Some characterizations of the spacelike, the timelike and the null curves on the pseudohyperbolic space $\mathbb{H}_{0}^{2}$ in $\mathbb{E}_{1}^{3}$," Kragujevac Journal of Mathematics, vol. 22, pp. 71-82, 2000.

[10] B. O’Neill, Semi-Riemannian Geometry, Academic Press, New York, NY, USA, 1983. 


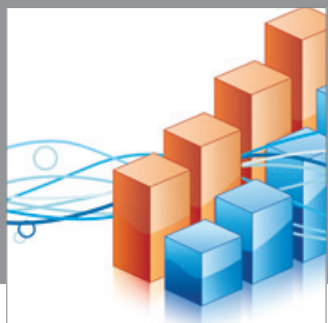

Advances in

Operations Research

mansans

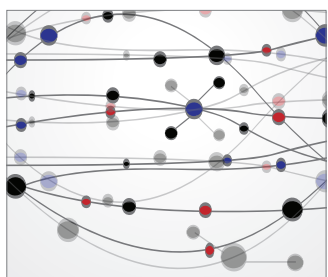

The Scientific World Journal
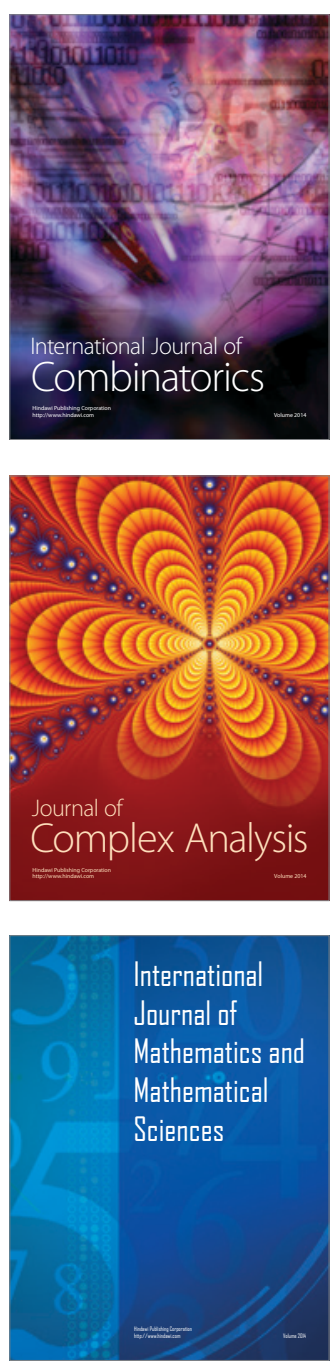
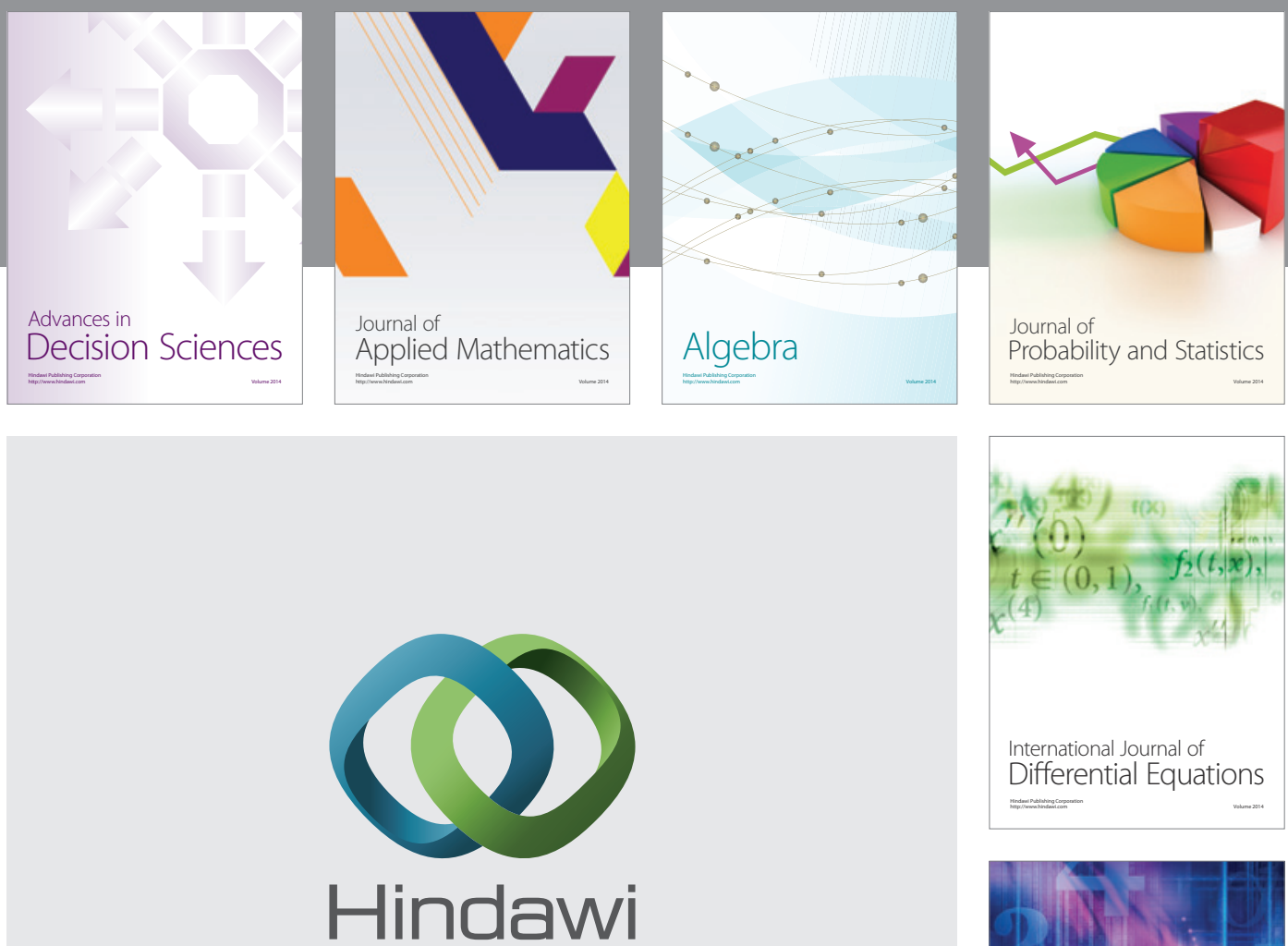

Submit your manuscripts at http://www.hindawi.com
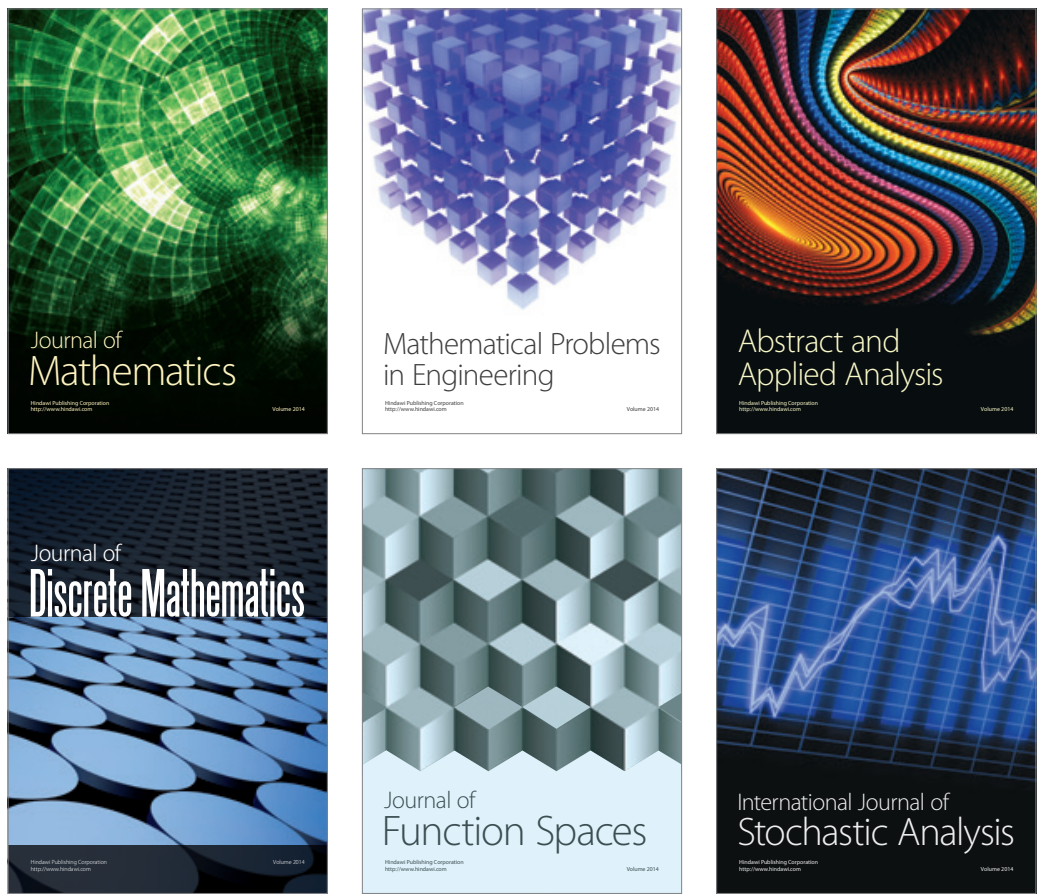

Journal of

Function Spaces

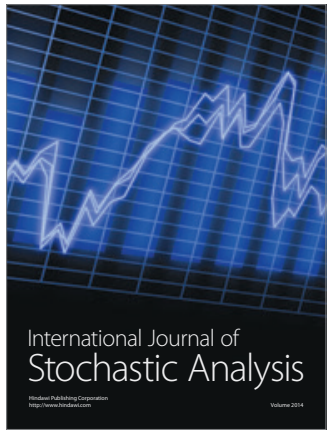

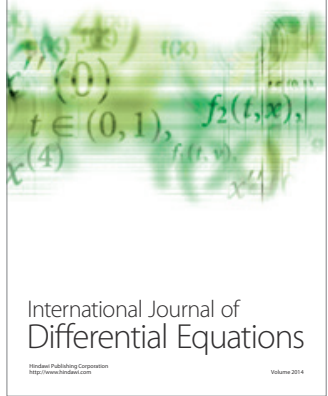
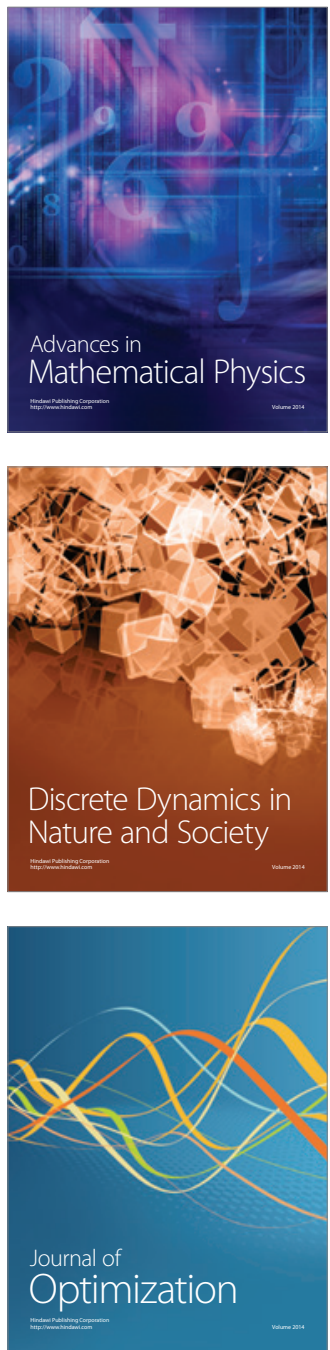